\title{
Investigating optical complexity of the phase transition in the intensity of a fibre laser radiation
}

\author{
Andrés Aragoneses ${ }^{\mathrm{a}}$, Laura Carpi ${ }^{\mathrm{b}}$, Nikita. Tarasov ${ }^{\mathrm{c}, \mathrm{d}}$, Dmitri. V. Churkin ${ }^{\mathrm{c}, \mathrm{e}, \mathrm{f}}$, \\ M. C. Torrent ${ }^{\mathrm{b}}$, Cristina Masoller ${ }^{\mathrm{b}}$, and Sergei. K. Turitsyn ${ }^{\mathrm{c}}$

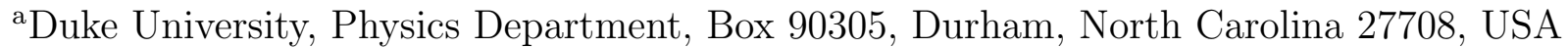 \\ bepartament de Física i Enginyeria Nuclear, Universitat Politècnica de Catalunya, 08222 \\ Barcelona, Spain \\ ${ }^{\mathrm{c}}$ Aston Institute of Photonic Technologies, Aston University, UK \\ dinstitute for Computational Technologies, SB RAS, Novosibirsk 630090, Russia \\ eInstitute of Automation and Electrometry SB RAS, Novosibirsk, Russia \\ ${ }^{\mathrm{f}}$ Novosibirsk State University, Russia
}

\begin{abstract}
Fibre lasers have been shown to manifest a laminar-to-turbulent transition when increasing its pump power. In order to study the dynamical complexity of this transition we use advanced statistical tools of time-series analysis. We apply ordinal analysis and the horizontal visibility graph to the experimentally measured laser output intensity. This reveal the presence of temporal correlations during the transition from the laminar to the turbulent lasing regimes. Both methods allow us to unveil coherent structures with well defined time-scales and strong correlations both, in the timing of the laser pulses and in their peak intensities.
\end{abstract}

Keywords: Complexity, fibre laser, chaos, delay systems, turbulence

\section{INTRODUCTION}

Typical fibre laser features nonlinear interactions of millions of longitudinal cavity modes in regimes far from thermal equilibrium. Therefore, resulting dynamics is rather complex [1-7], apart from the situations when multitude of modes are phase-locked forming coherent pulses effectively leading to reduced number of important degrees of freedom. In general, wave dynamics in fibre lasers is very complex and observed equilibrium is, typically, only statistical, as in other wave turbulence systems [7]. Though the underlying physical effect, nonlinear four-wave mixing, is purely deterministic, it has been realized that a dynamical description of such complex dynamics is not fully adequate approach and wave turbulence or kinetic theory techniques should be used. Entropy based analysis is appropriated in complex kinetic systems to study the dynamical complexity of light generation in fiber lasers. The role of temperature is played here by optical noise that occurs in the gain medium or by the effective non-linear noise due to four-wave-mixing induced mode dephasing.

Recently, the analogy between hydrodynamic transition to turbulence and change of operational regimes in fibre lasers has been studied both experimentally and theoretically [7,8]. A transition from highly ordered lasing regime to more irregular lasing, characterized by extreme, apparently random intensity fluctuations was reported. Such transition, being a relevant example of a phase transition in a one dimensional physical system, was shown to be accompanied by the occurrence of coherent spatio-temporal structures [7].

In this work we characterize the complex dynamics to find underlying correlations and/or specific time-scales in the easily measurable intensity fluctuations of laser radiation [8]. In order to investigate this issue we use two nonlinear analysis tools: ordinal analysis [9] and the horizontal visibility graph [10]. Both methods have been widely used to analyze the observed output signals of complex systems, being able to provide new information,

Further author information: (Send correspondence to A.A. or C.M.)

A.A.: E-mail: andres.aragoneses@phy.duke.edu

C.M.: E-mail: cristina.masoller@upc.edu

Semiconductor Lasers and Laser Dynamics VII, edited by Krassimir Panajotov, Marc Sciamanna,

Angel Valle, Rainer Michalzik, Proc. of SPIE Vol. 9892, 98920L · C) 2016 SPIE

CCC code: $0277-786 \mathrm{X} / 16 / \$ 18 \cdot$ doi: $10.1117 / 12.2225684$

Proc. of SPIE Vol. 9892 98920L-1 
not available with other methods, such as identification of frequent and/or missing patterns in the data, the classification of different behaviors, the characterization of deterministic and stochastic events, etc. [1118]. Here we show that they both provide consistent information, allowing to clearly identify the presence of long-range temporal correlations in the experimentally measured laser output intensity.

\section{THE EXPERIMENTS}

We measure an output temporal intensity dynamics of a quasi-CW Raman fiber laser formed of $1 \mathrm{~km}$ of normal dispersion fiber placed between two fiber Bragg gratings acting as cavity mirrors [7]. State of the art experimental capabilities allowed us to register long time traces with total number of intensity data points of 50 million. Taking into account the discretisation time of $12.5 \mathrm{ps}$, the intensity dynamics over $625 \mathrm{~s}$ could be captured. In order to be able to compare among time-series recorded at different pump power, each time-series is normalized to have zero-mean and unit variance. Depending on power, the generation regime can be considered as laminar or turbulent as was described in ref. [7]. The transition occurs at pump power $0.9 \mathrm{~W}$ (see [7] for details). Despite the radically different coherence properties of radiation in these two regimes, the output intensity, I(t), looks similar and irregular at all powers, as seen in Fig. 1, with typical intensity probability distribution function (pdf) of intensity values, $\mathrm{p}(\mathrm{I}(\mathrm{t}))$, shown on Fig. 1.

\section{THE TIME-SERIES ANALYSIS}

From experimentally measured intensity dynamics, I(t), we generate two new data sets. In order to consider only the extreme inten- sity fluctuations, we select an adequate threshold and filter out the intensity peaks whose height is below the threshold, keeping a sequence of intensity peak heights, $I_{\max , i}$, which are above the threshold, as shown in Fig. 1. Naturally, the precise definition of extreme fluctuations depends on the system under investigation. In hydrodynamics, when the height of a wave is larger by a factor of 3 than average, this wave is considered extreme, but in optics, fluctuations of much higher amplitudes compared to the average can often be observed. Here, because each time series is normalized to zero mean and unit variance, the thresholds used are in units of the standard deviation, $\sigma$ : with threshold 0 , the peaks considered are only those above the mean value; with threshold 1 , the peaks are only those above $\sigma$.

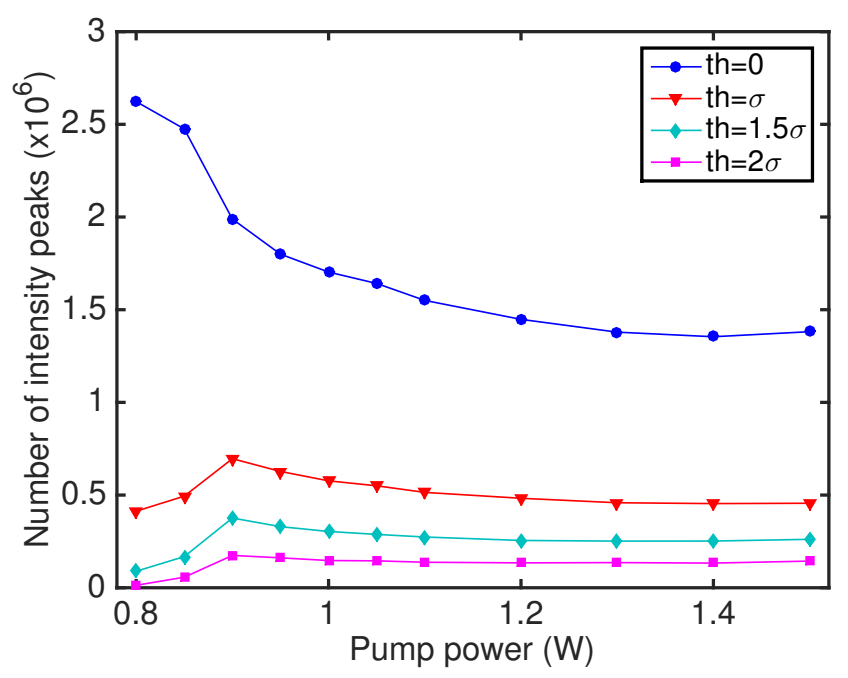

Figure 1. Number of events (peaks) versus pump power of the laser. There is a clear change in tendency at $\mathrm{P}=0.9 \mathrm{~W}$ which is captured for thresholds larger than $\sigma$.

The number of the peaks found in intensity dynamics measured at different pump power level is shown on Fig. 1 depending on the threshold value. We note that, if the threshold is low, the number of peaks decreases with the pump power, but if the threshold is high, the number of peaks increases with the pump power until 
$0.9 \mathrm{~W}$, where it is maximum, then, with further increase of the pump power, the number of peaks diminishes. The transition between two different generation regimes laminar and turbulent regime could be easily detected at $0.9 \mathrm{~W}$. This transition will be characterized by means of additional parameters. In the following we fix the threshold equal to 2, i.e., we analyze only the intensity peaks that are above $\sigma$. The robustness of the results with respect to the choice of the threshold is also shown (see figure 2). As each local intensity peak has also a time instant $T_{i}$ at which it occurs, we generate in parallel another data set: a sequence of time intervals between local intensity peaks, $\Delta t_{i}$.

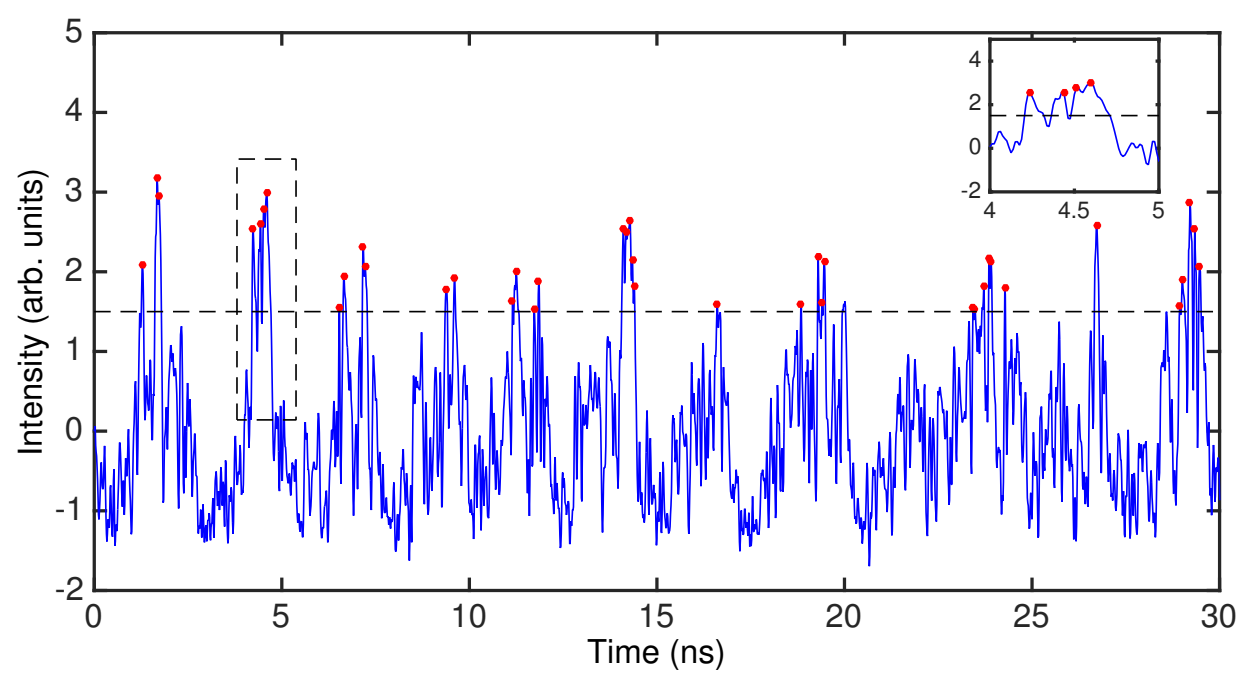

Figure 2. Stochastic dynamics of a quasi-cw Raman fiber laser: intensity time series measured at pump power $0.9 \mathrm{~W}$ (the dots indicate the peak values above a threshold, indicated with a dashed line, and the inset displays a detail)

We use two methods of time-series analysis to investigate the complexity of the dynamics and to uncover hidden temporal correlations. These methods ar known as ordinal analysis and horizontal visibility graph (HVG), which are represented schematically in Fig. 1. Ordinal analysis [9] transforms a time series $\left\{x_{i}\right\}$ (where $\left\{x_{i}\right\}$ can be either the sequence of peak heights, $I_{\max , i}$, or the sequence of time intervals between peaks, $\left.\left\{\Delta t_{i}\right\}\right)$ into a sequence of symbols (referred to as ordinal patterns, OPs), by considering the order relation among $\mathrm{D}$ values of the time-series. There are 2 different ordinary patterns of length 2: pattern 01 if $x_{i}<x_{i+1}$ and pattern 10 if $x_{i}>x_{i+1}$. If $\mathrm{D}=3$, there are 6 possible patterns: $x_{i}<x_{i+1}<x_{i+2}$ gives $012, x_{i+2}<x_{i+1}<x_{i}$ gives 210, etc. The number of possible patterns of the given length D is D!. This way, a sequence of patterns could be generated from the sequence of the peak heights or from the sequence of time intervals between the peaks.

Once the sequence of patterns is deffined, we calculate the probability to find the given pattern in the data set, $p_{i}$. The entropy computed from their probabilities, $p_{i}$, of occurrence in the time series, $S_{P E}=p_{i} \log \left(p_{i}\right)$, known as permutation entropy, has been shown to be an appropriated measure of the complexity of a timeseries $[9,12,13]$. When there are no serial correlations in the time-series $\left\{x_{i}\right\}$, then all the patterns are equally probable and $S_{P E}=\log D$ !. On the contrary, when there are serial correlations, then the OPs are not all equally probable, and the permutation entropy will be $S_{P E}<\log D$ !. In the following we refer to the normalized entropy, $S_{P E} / \log D$ !, as PE entropy. Thus, with an appropriate choice of pattern length $\mathrm{D}$, the OP probabilities and the $\mathrm{PE}$ entropy will capture the existence of underlying correlations in the time series.

To verify the presence of correlations independently, we also apply the HVG method [10] that converts a time series, $\left\{x_{i}\right\}$, into a graph by considering each data point, $x_{i}$, as a node. Any two nodes are connected by a link (or edge) if horizontal visibility exists between them: $x_{i}$ and $x_{j}$ are connected if it is possible to trace a horizontal line linking $x_{i}$ and $x_{j}$ not intersecting intermediate data; mathematically, this means that $x_{i}$ and $x_{j}$ are connected if: $x_{i}, x_{j}>x_{n}$ for all $i<n<j$. This graph representation of the time series $\left\{x_{i}\right\}$ takes into account both, the order and the values of the data points. Time series with different dynamics are mapped into 
graphs that exhibit distinct topological structures [14]. The topology of a graph is characterized by the degree distribution, $\mathrm{p}(\mathrm{k})$, that is the probability that a node has $\mathrm{k}$ links. Thus, the entropy of the degree distribution, $S_{H V G}=p_{k} \log \left(p_{k}\right)$ (in the following, referred to as HVG entropy), is another measure of the complexity of the time series $\left\{x_{i}\right\}$ [18]. An appropriate normalization of the HVG entropy is that of the Gaussian white noise, which rapidly converges to stable values for increasing time series lengths (percentage variations for times series with $N=10^{5}$ and $N=5 \times 10^{5}$ data points are $10^{4}$ and $10^{5}$; in contrasts, the normalization through $\log (\mathrm{N})$ results in decreasing entropy values as $\mathrm{N}$ increases [18]). Thus, in the following, the HVG entropy is normalized to the entropy of Gaussian white noise. The HVG method also allows to analyze different time-scales by constructing the graph not from all the raw data points, but from lagged data: $\left\{x_{i}, x_{i+\tau}, x_{i+2 \tau}, \ldots\right\}$.

The two analysis methods used transform the time series $\left\{x_{i}\right\}$ into a sequence of integer numbers, $\left\{k_{i}\right\}$ (in the OP case, $k_{i} \epsilon[1, D !]$ is the pattern label: if $01, k_{i}=1$; if $10, k_{i}=2$, etc.; in the HVG case, $k_{i} \epsilon[1, N 1]$ is the degree of $x_{i}$, with $\mathrm{N}$ being the number of data points in the time series), they have important differences: while the OP method requires the pre-definition of the length of the pattern $\mathrm{D}$, and does not take into account the values of the data points, the horizontal visibility graph method does not require to pre-define an analysis length, and considers both, the order relation and the actual values of the data points.

Figure 3 displays the probabilities of the six patterns of length 3 calculated from the sequence of intensity peaks (a) and from the sequence of the time intervals (b), respectively. The error bars are computed with a binomial test, and indicate a confidence level of 95\%; the gray region indicates probability values that are consistent with a uniform distribution (i.e., consistent with no serial correlations in the data) [19]: it indicates probabilities in the interval $p \pm 3 \sigma$, where $p=1 / D$ ! and $\sigma=\sqrt{(}(1 p) / N)$ with $\mathrm{D}$ ! being the number of possible patterns and $\mathrm{N}$ being the number of ordinal patterns.
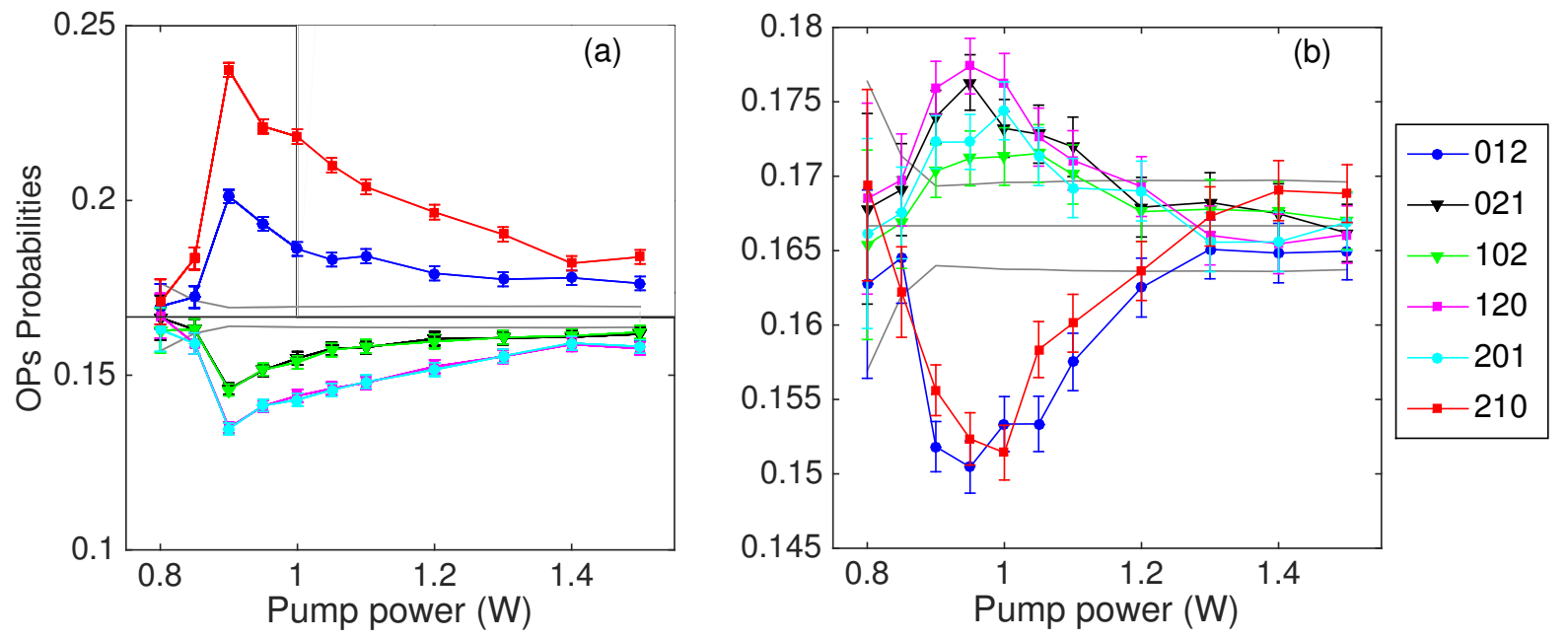

Figure 3. Probabilities of ordinal patterns of length $\mathrm{D}=3$ vs pump power calculated from the sequence of (a) intensity peaks and (b) time intervals between consecutive peaks. The error bars are computed with a binomial test and the gray lines indicate probability values consistent with the uniform distribution.

It can be observed that the variation of the probabilities with the pump power captures the transition between two dynamical regimes: below the transition the OPs are equally probable, while during the transition from laminar to turbulent regime their probabilities are different from equiprobability. It can also be noticed that, during the transition, the patterns 012 and 210 become more probable (less probable) when the OPs are calculated from the $I_{\max , i}$ sequence (from the $\left\{\Delta T_{i}\right\}$ sequence). We also note that the patterns calculated from the intensity peaks capture more determinism than those computed from the time intervals [notice the difference in the vertical scales of Figs. 2(a) and 2(b)]. This indicates that the timing of the high intensity peaks is more random than their peak values. 
The PE-entropy, Fig. 3(a), quantifies this effect by decreasing sharply at the transition power. A similar behavior is observed when computing the HVG-entropy, Fig. 3(b). In both cases, the entropy values computed from surrogate (shuffle) data are also indicated, and one can observe that, as expected, the transition is not detected in the shuffle data.

In Fig. 4(a) the PE-entropy was computed from $\mathrm{D}=3$ OPs; similar plots were obtained with $D \epsilon[26]$. Larger $\mathrm{D}$ values were not considered due to the finite length of the dataset: while the raw intensity time series contains 50 million data points, the number of high intensity peaks (above $2 \sigma$ ), as shown in Fig. 1 , is about $10^{5}$, depending on the pump power.
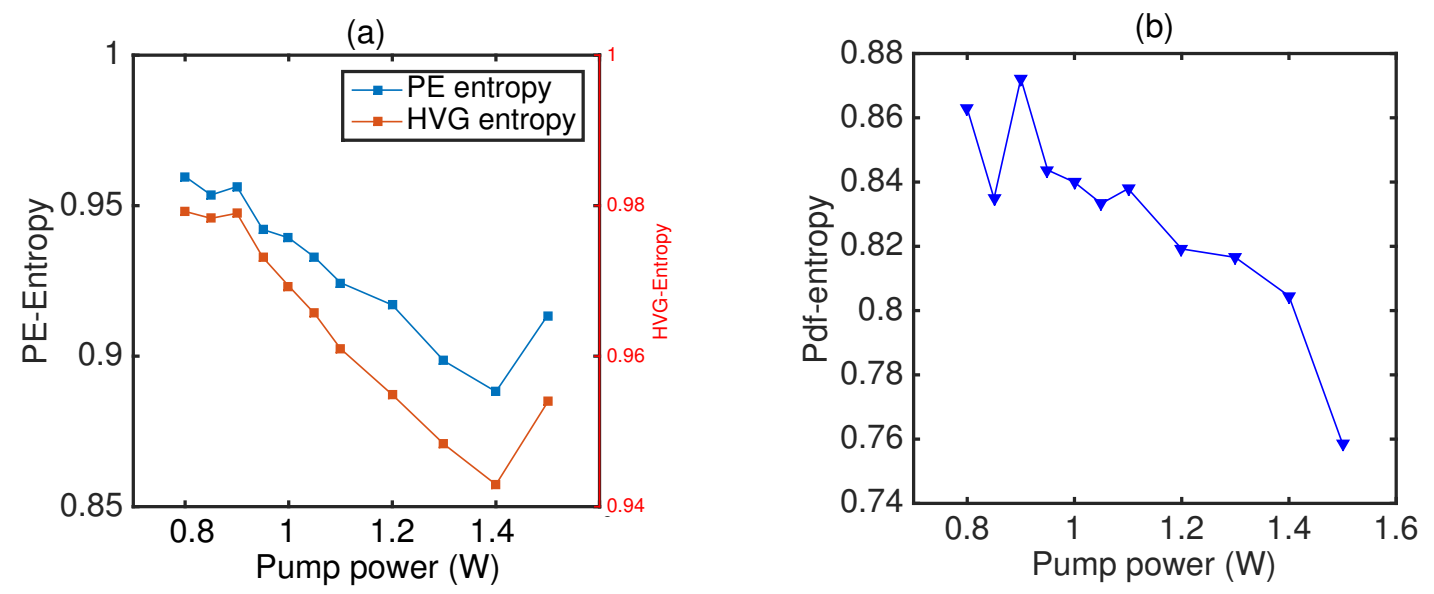

Figure 4. (a) Permutation entropy and HVG entropy vs the pump power. Calculations are performed with D 143 OPs. (b) PDF entropy calculated from the distribution of intensity values.

The probabilities of patterns 012 and 210 provide a measure of the persistence of the time-series, i.e., the probability that the sign of $x_{i}-x_{i-1}$ persists in the next step [11]. Thus, at the transition, if there are two consecutive peaks with increasing height, the next peak is likely to be larger than the previous one (and if there are two consecutive peaks with decreasing height, the next one is likely to be smaller than the previous one); on the contrary, in the sequence of time-intervals, two consecutive intervals that are increasingly long $\left(\Delta T_{i}<\Delta T_{i+1}\right)$ are likely to be followed by shorter interval $\left(\Delta T_{i+1}>\Delta T_{i+2}\right)$, and two consecutive decreasing intervals $\left(\Delta T_{i}>\Delta T_{i+1}\right)$ are likely to be followed by a longer one $\left(\Delta T_{i+1}<\Delta T_{i+2}\right)$.

Let us note that the optimal value of pattern length $D$ depends of the length of correlations embedded into the time series [9]. We note however that the OP sequence does not take into account the actual values of the data points $\left\{x_{i}\right\}$ and thus, does not provide information about the presence of extreme fluctuations in the time-series. By considering lagged data points, this method allows to analyze specific time-scales. For example, correlations among $\left(x_{i}, x_{i+\tau}, x_{i+2 \tau}\right)$ can be inferred from the corresponding OP probabilities.

In order to demonstrate the robustness of the results with respect to the choice of the threshold, in Fig. 5 we plot the OP probabilities with $D=2$ and $D=3$ and the PE-entropy for three thresholds: 0,1 , and 2 (i.e., as the time-series are normalized to zero mean and unit standard deviation, with these thresholds we consider only the intensity peaks that are above the mean value, above one standard deviation, and above two standard deviations). In the three cases one can observe that the same transition is detected, at the same value of the control parameter.

In the case of ordinal analysis, as Fig. $3^{* * *}$ shows, the results are robust also with respect to the length of the ordinal pattern, $D$.

To investigate the presence of specific time-scales in the dynamics, we analyze the lagged time series, i.e. the sequence of $\left\{I_{i}, I_{i+\tau}, I_{i+2 \tau}, \ldots\right\}$, where $\tau$ is the lag time. We begin by considering the case $\tau=1$ (i.e., we analyze all data points). Figure 3(a) displays the PE entropy and the HVG entropy vs the pump power, and same behavior is seen in both entropies: there is a clear transition at pump power $0.9 \mathrm{~W}$, where both entropies 

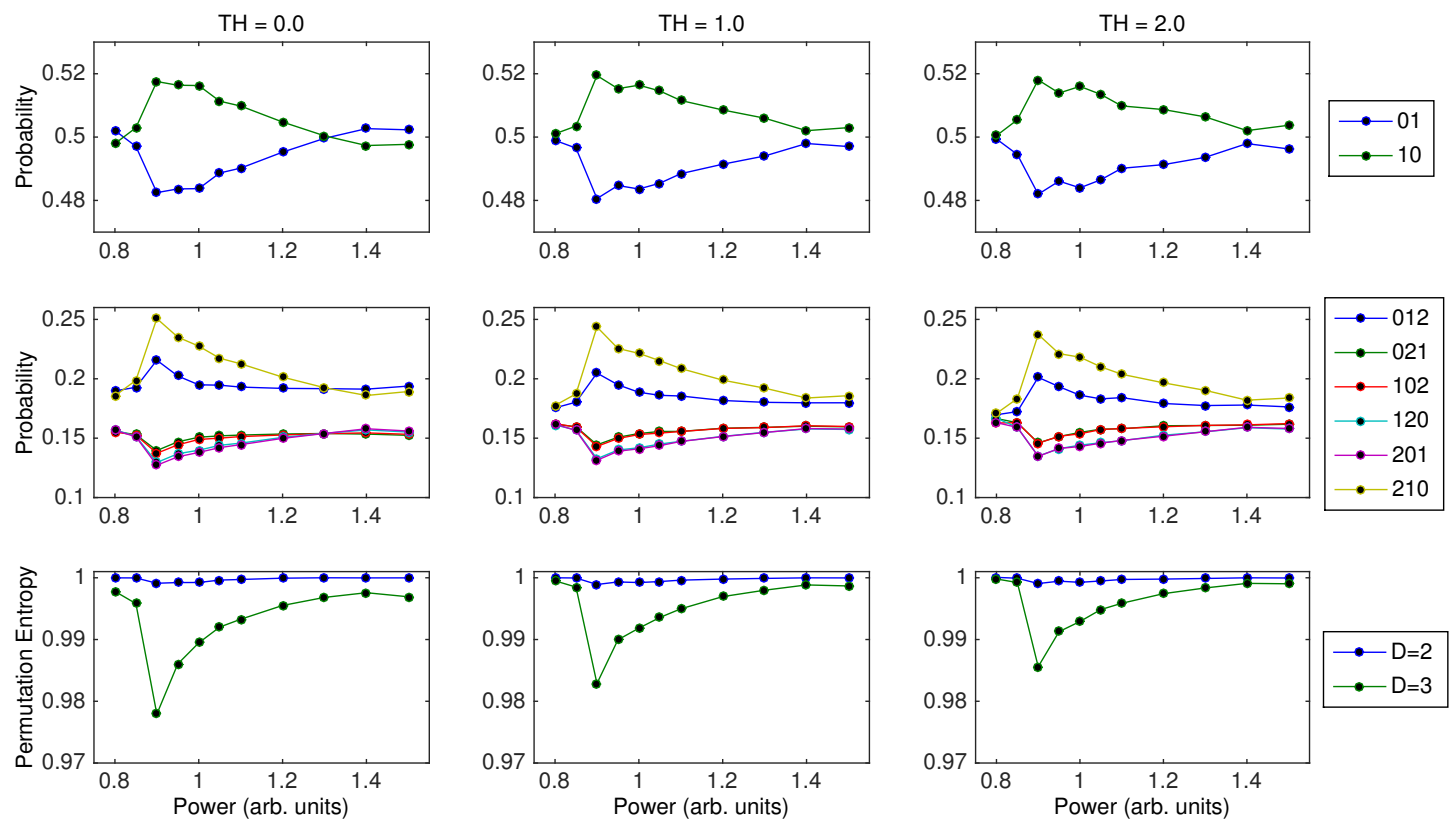

Figure 5. (a) OPs for $\mathrm{D}=2$ (first row) and $\mathrm{D}=3$ (second row) for different thresholds $(0, \sigma, 2 \sigma$. PE entropy is plotted for the same thresholds in the last row.

smoothly decrease. It is also observed that for the highest pump power, both entropies increase again. This reveals that during the transition there is an increase in the "ordering" of consecutive intensity values (that is captured by both entropies, which decrease), but for the highest pump power the trend reverses and the disorder increases. In contrast, the entropy computed in the conventional way and referred as pdf-entropy (i.e. the entropy calculated from the intensity pdfs of the initial intensity dynamics, $I(t)$ ) does not capture this behavior: as it can be seen in Fig. 3 (b), after the transition the pdf-entropy monotonously decreases with the pump power. Thus, the PE and HVG entropies provide consistent information, which complements that gained from the standard pdf-entropy. The good agreement between the two entropies, also seen in Figs. 2(c) and 2(d), is remarkable because the two methods transform a time series into a sequence of integer numbers by using very different encoding rules.

By varying the value of the lag time $\tau$, i.e. by taking into account not all points in data sets, but every second $(\tau=2)$ point, every third $(\tau=3)$ point etc., we are able to identify a specific oscillation time-scale in the intensity time-series during the transition. The PE entropy vs $\tau$ for pump powers below $(0.85 \mathrm{~W})$, at $(0.90 \mathrm{~W})$ and above $(0.95 \mathrm{~W})$ the transition is displayed in Fig. 6. Here we can notice that, at the transition, there are specific lags for which the PE entropy decreases sharply. Similar results were obtained with the HVG entropy.

The sharp minima indicate that, for pump power $0.90 \mathrm{~W}$ and for specific lags, 6 different patterns of length $D=3$ are not all equally probable, and thus, there are serial correlations in the sequence of lagged intensity values. To explore the length of such correlations, we computed the PE entropy using longer ordinary patterns ( $D=4$ and $D=6)$ and found that the minima were more pronounced, revealing the existence of long serial correlations. .

To investigate the nature of these correlations we plot on Fig. 7 how the probabilities of 6 different ordinary patterns of length $D=3$ depend on the lag time. We note a periodic alternation in which 012 and 210 became the more probable or the less probable patterns. The probabilities of the other four patterns are similar (no clear clusters are seen). The lag values for which 012 and 210 are less probable correspond, as expected, to the lag values where the autocorrelation function is minimum (and negative). However, unexpectedly, the lag values for which 012 and 210 are more probable, do not correspond to the maxima of the autocorrelation; and moreover, 


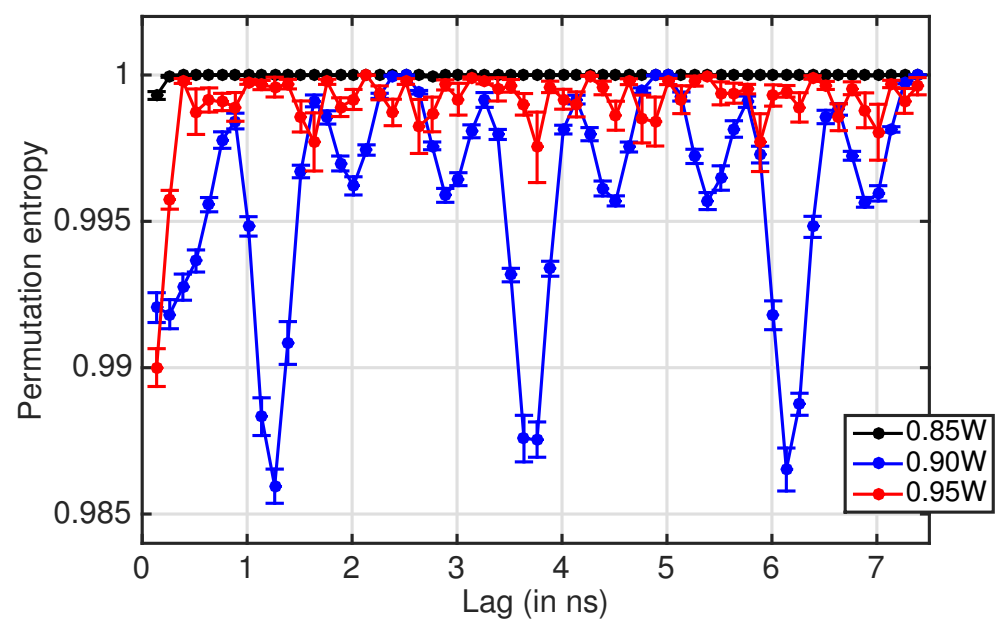

Figure 6. Permutation entropy vs the lag time before (0.85), at (0.9), and after (0.95 W) the transition to optical turbulence. Error bars were computed by dividing the data in 10 windows and computing the PE entropy in each window.

for the lag values where the autocorrelation is maxima, the all six ordinary patterns have similar probabilities. These observations suggest that ordinal analysis identifies subtle correlations in the ordering of data points, which are not seen by the standard autocorrelation function, that measures correlations in the values of the data points.

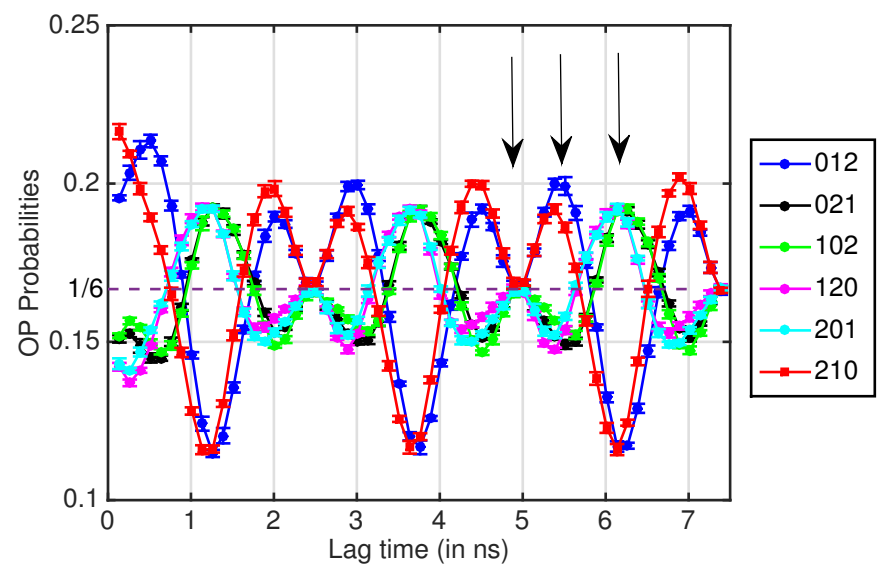

Figure 7. Probabilities of the ordinary patterns of length $\mathrm{D}=3$ vs lag time $\tau$.

These "order correlations" result into different types of spatio-temporal patterns. Let us recall that the initial intensity spatio-temporal dynamics could be seen as intensity spatio-temporal dynamics [8]. Similar concept has been recently employed for studying interaction of cavity solitons and topological solitons as addressable bits [22]. Here we apply this concept and choose specific lag times defined from Fig.7 to be used as round-trip times in processing of the initial data series (see [8] for details of plotting spatio-temporal dynamics). The lag times chosen correspond to where the six patterns are equally probable, when '210' and '012' are more probable than the rest and when they are less probable than the rest, indicated in Fig.7 with arrows.

Figures 8(a)-(c) display examples with lags such that patterns 012 and 210 are as probable [Fig. 8(a)], more probable [Fig. 8(b)]) and less probable [Fig. 8(c)] than the other four patterns. We can see that these spatio- 
temporal dynamics of patterns display clear and different coherent structures. These observations can be useful for confronting the predictions of state-of-the-art laser models with empirical data, and the theoretical studies could provide insight into the physical mechanisms underlying these correlations.
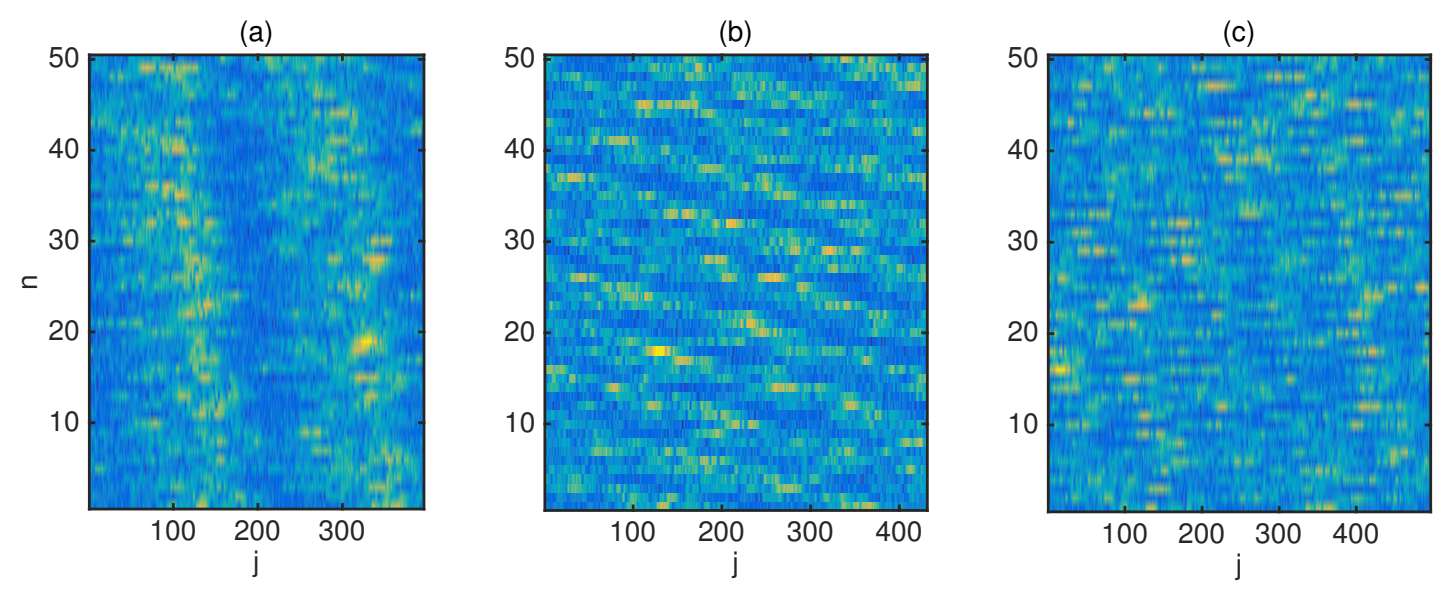

Figure 8. Spatiotemporal structures identified with the specific lags indicated with arrows in panel (a). The color scale indicates the value of Ii with i $14 \mathrm{n} \mathrm{j}$ and 14396,431 , and 496 in units of the sampling time. The pump power is $0.9 \mathrm{~W}$.

To summarize, by applying two independent tools of nonlinear time-series analysis we have uncovered longrange temporal correlations in the intensity output of a fibre laser during the transition to a wave turbulence regime. Output of laser radiation is easily measurable, making these easily implementable methods useful and valuable techniques for investigating coherent structures in complex laser radiation. Both approaches can be applied to any high-dimensional complex systems that undergo similar transitions accompanied by the generation of extreme fluctuations.

\section{ACKNOWLEDGMENTS.}

We acknowledge support from the Spanish MINECO (FIS2012-37655-C02-01), European Oï $\neg$ fce of Aerospace Research and Development (EOARD) (Grant No. FA9550-14-1-0359), CNPq Brazil, the ERC project UL-TRALASER, the Russian Ministry of Education and Science ( 14. B25.31.0003, 14.584.21.0014), H2020 project CARDIALLY, the Russian Foundation for Basic Research (15-02-07925, 16-32-60153). N. T. is supported by the Russian Science Foundation (Grant No. 14-21-00110).

\section{REFERENCES.}

[1] C. J. S. de Matos, L. de S. Menezes, A. M. Brito-Silva, M.A. Martinez Gamez, A.S.L. Gomes, and C.B. de Araujo, Phys. Rev. Lett. 99, 153903 (2007).

[2] S. K. Turitsyn, J. D., Ania-Castanon, S. A. Babin, V. Karalekas, D. Churkin, S. I. Kablukov, A. E. El-Taher, E. V. Podivilov, and V. K. Mezentsev, Phys. Rev. Lett. 103, 133901 (2009).

[3] S. K. Turitsyn, S. A. Babin, D. V. Churkin, I. D. Vatnik, M. Nikulin, and E. V. Podivilov, Phys. Rep. 542,133 (2014).

[4] A. El-Taher, O. Kotlicki, P. Harper, S. Turitsyn, and J. Scheuer, Laser Photonics Rev. 8, 436 (2014).

[5] D. V. Churkin, A. E. El-Taher, I. D. Vatnik, Castanon, P. Harper, E. V. Podivilov, S. A. J. D. Ania-Babin, and S. K. Turitsyn, Opt. Express 20, 11178 (2012).

[6] A. Picozzi, J. Garnier, T. Hansson, P. Suret, S. Randoux, G. Millot, and D. N. Christodoulides, Phys. Rep. 542, 1 (2014). 
[7] E. G. Turitsyna, S. V. Smirnov, S. Sugavanam, N. Tarasov, X. Shu, S. A. Babin, E. V. Podivilov, D. V. Churkin, G. E. Falkovich, and S. K. Turitsyn, Nat. Photonics 7, 783 (2013).

[8] A. Aragoneses, L. Carpi, N. Tarasov, D. V. Churkin, M. C. Torrent, C. Masoller, and S. K. Turitsyn, Phys. Rev. Lett. 116, 033902 (2016).

[9] C. Bandt and B. Pompe, Phys. Rev. Lett. 88, 174102 (2002).

[10] B. Luque, L. Lacasa, F. Ballesteros, and J. Luque, Phys. Rev. E 80, 046103 (2009).

[11] C. Bandt and F. Shiha, J. Time Ser. Anal. 28, 646 (2007).

[12] M. Zanin, L. Zunino, O. A. Rosso, and D. Papo, Entropy 14, 1553 (2012).

[13] J. M. Amigo et al., Eur. Phys. J. Spec. Top. 222, 241 (2013)

[14] L. Lacasa and R. Toral, Phys. Rev. E 82, 036120 (2010).

[15] J. B. Elsner, T. H. Jagger, and E. A. Fogarty, Geophys. Res. Lett. 36, L16702 (2009).

[16] M. Ahmadlou, H. Adeli, and A. Adeli, Journal of neural transmission 117, 1099 (2010).

[17] U. Parlitz, S. Berg, S. Luther, A. Schirdewan, J. Kurths, and N. Wessel, Computers in Biology and Medicine 42, 319 (2012).

[18] M. Gomez Ravetti, L. C. Carpi, B. Goncalves, A. C. Frery, and O. A. Rosso, PLoS One 9, e108004 (2014).

[19] A. Aragoneses, S. Perrone, T. Sorrentino, M. C. Torrent, and C. Masoller, Sci. Rep. 4, 4696 (2014).

[20] D. V. Churkin, S. Sugavanam, N. Tarasov, S. Khorev, S. V. Smirnov, S. M. Kobtsev, and S. K. Turitsyn, Nat. Commun. 6, 7004 (2015).

[21] J. K. Jang, M. Erkintalo, S. Murdoch, and S. Coen, Nat. Photonics 7, 657 (2013).

[22] B. Garbin, J. Javaloyes, G. Tissoni, and S. Barland, Nat. Commun. 6, 5915 (2015). 